\title{
Morfometría macroscópica del cuerpo lúteo de vacas cebú gestantes y no gestantes en el trópico colombiano
}

\section{Macroscopic morphometry of the corpus luteum of pregnant and non-pregnant zebu cows in the Colombian tropics}

González T Marco* M.Sc; De La Rosa T Eduardo² MVZ; Mendoza M Carlos² MVZ;

'Universidad de Córdoba, Facultad de Medicina Veterinaria y Zootecnia.

Departamento de Ciencias Pecuarias. Montería, Colombia. Instituto de Investigaciones Biológicas del Trópico (IIBT).

${ }^{2}$ Practica Privada.

\section{Keywords:}

Anatomical planes;

corpus luteum;

ovaries;

shape of the corpus luteum; weight of the corpus luteum.

\section{Abstract}

The objective of the study was to determine the volume, weight, measures, ovarian location and shape of the corpus luteum of pregnant and non - pregnant cows from zebu cows of the Colombian tropics. 528 reproductive tracts were collected; 264 pregnant and 264 non-pregnant of cows benefited at the local slaughterhouse in Monteria, Córdoba, Colombia. The period of collection of the samples was extended for three months. After collection of each reproductive tract, the ovaries were separated, identified as right and left, weighed and measured. Then the drawing of the location of the corpus luteum was performed on the ovary according to the anatomical planes previously established in the corresponding form. Subsequently the corpus luteum was removed to perform their measurements, weighings and visualization of their shape. There were statistical differences between the locations of the corpus luteum in the ovary: Anterior pole, posterior pole, free edge, upper face and lower face $(p \leq 0.05)$. The weight and volume of gestational corpus luteum was greater by 30 and $27.9 \%$ than the corpus luteum of non-pregnant cows. The predominant form por shape of the corpus luteum in both pregnant and non-pregnant cows was oval, then pyramidal and finally rounded. No gestation was observed contralateral to the location of the corpus luteum.

\section{Resumen}

El objetivo del trabajo fue determinar el volumen, peso, medidas, ubicación ovárica y forma del cuerpo lúteo de vacas gestantes y no gestantes de vacas del trópico colombiano. Se recolectaron 528 tractos reproductivos; 264 gestantes y 264 no gestantes de vacas beneficiadas en el frigorífico local de Montería, Córdoba, Colombia. El periodo de recolección de las muestras se extendió por tres meses. Después de la recolección de cada tracto reproductivo se separaron los ovarios, identificándolos como derechos e izquierdos, se pesaron y se midieron. Luego se realizó el dibujo de la ubicación del cuerpo lúteo en el ovario de acuerdo con los planos anatómicos previamente establecidos en el formulario correspondiente. Posteriormente se removieron los cuerpos lúteos para realizar sus medidas, pesajes y visualización de su forma. Hubo diferencias estadísticas entre las ubicaciones del cuerpo lúteo en el ovario: Polo anterior, polo posterior, borde libre cara superior y cara inferior $(\mathrm{p} \leq 0.05)$. El peso y volumen del cuerpo lúteo gestacional fue mayor en un 30 y $27.9 \%$ que el cuerpo lúteo de vacas no gestantes. La forma predominante del cuerpo lúteo tanto en vacas gestantes como en no gestantes fue la ovalada, luego la piramidal y por último la redondeada. No se observó ninguna gestación contralateral a la ubicación del cuerpo lúteo. 


\section{Introducción}

El aparato genital femenino de los mamíferos durante el período de actividad genital sufre modificaciones estructurales que se reproducen siempre en el mismo orden y se repiten a intervalos periódicos según unritmo característico. Estas modificaciones, conocidas como ciclo estral comienzan con la pubertady prosigue durante toda la vida sexual, interrumpiéndose solamente durante la gestación. La duración del ciclo estral en la vaca es de 21 días y en las novillas de 20, aunque los ciclos e ntre 17 y 24 días son normales. El ciclo estral comprende cuatro fases que son: Proestro, Estro, Metaestro, Diestro (DERIVAUX, 1961, GARCIA, 1995).

El estro tiene una duración relativamente corta y oscila entre 12 y 24 horas, aunque con grandes fluctuaciones. Se caracteriza por una serie de cambios endocrinos que dan lugar a cambios fisiológicos, morfológicos y de comportamiento en las hembras. Normalmente suele señalarse como día cero (0) el del estro, ya que la ovulación se produce de manera espontánea unas horas después de finalizar éste. Los cambios endocrinos, una vez que se produce la primera ovulación, se suceden ordenadamente en una secuencia temporal definida y se inicia la vida media del cuerpo lúteo (GARCIA, 1995).

El proestro es la primera fase del ciclo y tiene una duración de 3-4 días y es donde se da el crecimiento del folículo de Graff, el cual entre otras, estradiol que vía sanguínea alcanza el oviducto, preparándolo para el transporte del óvulo hacia el útero. En esta etapa del ciclo estral también hay un engrosamiento de las paredes de la vagina con el fin de evitar lesiones durante el coito (DERIVAUX, 1961, Mc DONALD, 1981).

Después de 2 o 3 días de proestro, aparece el estro que tiene una duración de 14 a 18 horas (Mc DONALD, 1981). Esta etapa es conocida como celo, en la cual la hembra es receptiva al macho. Aquí se completa maduración del folículo de Graff, su dehiscencia y expulsión del óvulo, junto con el líquido folicular y células de la granulosa (ARTHUR, 1991).

El período después del estro es el metaestro y tiene una duración probable de 2 días (Mc DONALD, 1981). En esta fase se reorganiza el antro del folículo de Graff. La teca interna y los gránulos que no fueron expulsados forman el cuerpo lúteo. Este es un cuerpo sólido y de color amarillento, cuya función es la de producir progesterona principalmente. También impide la maduración del nuevo folículo de Graff, colabora con la implantación del óvulo fecundado y su nutrición al inicio de la preñez, y además, está íntimamente relacionado con la evolución de la glándula mamaria (DERIVAUX, 1961).

El diestro que sigue al metaestro se constituye en la etapa más larga del ciclo estral y tiene una duración de 279 días si hay gestación y de 17 días si empieza un nuevo ciclo estral (Mc DONALD, 1981). En esta etapa el cuerpo lúteo se desarrollado plenamente y ejerce un efecto marcado sobre la pared uterina (DERIVAUX, 1961).

Los ovarios son órganos pares, casi simétricos, contenidos en el abdomen, envueltos por el peritoneo, detrás del riñón correspondiente y normalmente, situados próximos al nivel de la tuberosidad ilíaca (BONADONNA, 1961). Suelen tener forma elipsoidal, algo deprimida en sentido lateral. El margen dorsal, o margen mesovárico o hilio del ovario, está adherido al mesovario, mientras que el ventral es libre. El polo craneal o anterior es denominado también polo libre. El posterior o caudal se llama así mismo polo uterino (BONADONNA, 1961).

Los ovarios en la vaca tienen un diámetro de 3 a $5 \mathrm{~cm}$ y un peso de 14 a $17 \mathrm{~g}$. Son ovalados y aplanados en sentido transversal. El tamaño de los ovarios depende fundamentalmente del período del ciclo estral en que es examinado y de sí contiene, o no, un cuerpo lúteo activo. La presencia de folículos no modifica tanto el tamaño del ovario (ARTHUR, 1991). Los ovarios de las vacas multíparas no difieren mucho de los de la novilla o vaca primípara; sin embargo, la mayoría de las veces son más grandes. Este incremento de tamaño es debido en parte a la progresiva deposición de tejido cicatrizal como resultado de su prolongada funcionalidad (ARTHUR, 1991).

La descripción anatómica del ovario incluye la enumeración y definición de las regiones tales como polos, superficies y bordes. Hay dos polos; anterior y posterior; dos superficies; una medial y otra lateral. Asimismo tiene dos bordes; fijo y libre. La denominación "borde fijo" se refiere al borde que está fijo al mesoovario. El opuesto es el "borde libre". La terminología utilizada para describir polos y superficies está basada en las relaciones de las gónadas durante el período embriológico. El eje mayor del ovario embrionario que cuelga libremente, es paralelo al eje del embrión y la terminología se explica por sí misma. Durante el desarrollo fetal posterior, los ovarios descienden a ocupar la posición en la que se encontrarán posteriormente en el animal maduro (FRANDSON y SPURGEON, 1995).

El cuerpo lúteo representa un órgano bastante voluminoso y alcanza más de la mitad del tamaño del ovario. La parte exterior que sobresale de la superficie 
del ovario, forma un botón sólido y frágil y puede tener hasta 2 centímetros de alto y 1.3 -2 centímetros de ancho. El cuerpo amarillo puede cambiar no sólo el tamaño del ovario sino también su forma, que depende de su localización. Por ejemplo, cuando el cuerpo amarillo se desarrolla en un polo ovárico, el ovario toma una forma más prolongada (de tabaco), cuando se desarrolla en su parte media puede tomar forma triangular, de una Y o de una L (HOLY, 1987).

Por razones desconocidas los dos ovarios no funcionan de manera idéntica en la mayor parte de especies domésticas. Se ha especulado en el sentido que el aporte sanguíneo al ovario quizá influya en la cantidad de gonadotropinas que llegan al mismo, las que a su vez dictan la función de estos órganos. (Mc DONALD, 1981). Existen numerosas pruebas que indican que en la vaca, el ovario derecho es más activo que el ovario izquierdo, como lo muestran los informes de Fincher, Erdheim, Arthur, Belling y otros, citados por ROBERTS (1979). En estos informes indicaron que alrededor del $60 \%$ de las preñeces ocurren en el cuerno derecho y el $40 \%$ en el izquierdo.

El cuerpo lúteo se desarrolla después la ovulación. En la pared interna del folículo se forman pliegues macroscópicos y microscópicos que penetran en la cavidad central. Estos pliegues están formados por un corazón central de estroma y grandes vasos sanguíneos que se distienden. Las células surgen pocos días antes de la ovulación y sufren una rápida regresión dentro de las 24 horas después de la ovulación, y las células de teca restantes se encuentran en un estado avanzado de degeneración (FRANDSON y SPURGEON, 1995).

El aumento en el peso del cuerpo lúteo al inicio es rápido. En general, el período de crecimiento es un poco más largo que la mitad del ciclo estral. En la vaca, el peso y el contenido de progesterona del cuerpo lúteo aumentan rápidamente entre el tercero y el décimo segundo día del ciclo y se mantiene más o menos constante hasta el día 16, cuando empieza la regresión (FRANDSON y SPURGEON, 1995). Si no hay fecundación el cuerpo lúteo sufre regresión y permite que maduren otros folículos ováricos más grandes. A medida que estas células degeneran, el órgano completo disminuye de tamaño, se vuelve blanco o café pálido y se llama entonces corpus albicans. Los cambios regresivos consisten en un engrosamiento de las paredes de las arterias en el cuerpo lúteo, una disminución en la·granulación citoplasmática, redondeo de la línea celular externa y vacuolación periférica de las grandes células del cuerpo lúteo (FRANDSON y SPURGEON, 1995).

El cuerpo lúteo del ciclo estral del bovino empieza a desaparecer 14 a 15 días después del estro y su tamaño disminuye a la mitad en unas 36 horas (FRANDSON y SPURGEON, 1995). Uno de los cambios morfológicos y fisiológicos más interesantes que ocurren durante el ciclo es la formación del cuerpo lúteo en el lugar del folículo después de su ruptura. En un tiempo relativamente corto las células que segregaban estrógenos durante la fase folicular se transforman en células luteales que segregan progesterona; ambas hormonas tienen un gran parecido químico, pero son fisiológicamente muy distintas (HANSEL and BLAIR, 1996).

El cuerpo lúteo quístico se diferencia fácilmente de los otros tipos. Prácticamente todas las estructuras son idénticas a las encontradas en el cuerpo amarillo normal. La característica diferencial decisiva es la sensación de fluctuación dentro de un cuerpo amarillo de aspecto normal. Se observa más frecuentemente en el postparto de las vacas y al parecer no influye en la función reproductora del animal (ZEMJANIS, 1980).

Con raras excepciones, el cuerpo lúteo gestacional se encuentra ubicado en el ovario correspondiente al cuerno del útero que contiene el feto; según Perkins, Olds y Seath, citados por ROBERTS (1979), menos del $2 \%$ de los cuerpos lúteos de gestación se encontraban en el ovario opuesto al cuerno que contenía al feto Erdheim, citado por ROBERTS (1979), informó solo un caso de este tipo en 3824 vacas preñadas. Belling, citado por ROBERTS (1979) encontró que la incidencia era del $0.52 \%$ mientras que Roberts y Setterghen, han observado esta peculiaridad en 1 de cada 3000 a 4000 vacas preñadas, lo que corresponde a $0.03 \%$ aproximadamente (ROBERTS, 1979).

En la teriogenología veterinaria no existen datos específicos sobre la morfología y características macroscópicas del cuerpo lúteo de hembras cebuínas (Bos indicus) del trópico bajo. La información que se tiene corresponde a ganado europeo (Bos taurus), y lo que se sabe acerca del ganado del trópico (Bos indicus), es prácticamente nulo.

Para el Médico Veterinario que ejerce la teriogenología veterinaria es importante conocer la determinación de la posición anotómica y espacial del cuerpo lúteo en el ovario de vacas no gestantes al momento de llevar acabo un examen clínico completo del estado general del tracto reproductivo.

El propósito del presente trabajo fue determinar la posición anatomo-espacial del cuerpo lúteo en los ovarios de vacas gestantes, así como determinar su peso, forma y volumen tanto del cuerpo lúteo como de los ovarios de las vacas cebuínas de la región Caribe. 


\section{Materiales y métodos}

Tipo de estudio. Se realizó un estudio de tipo descriptivo de corte transversal, en donde se examinaron tractos reproductivos gestantes y no gestantes de hembras bovinas cebuínas donados por el frigorífico Frigosinú S.A., de Montería, Córdoba, Colombia.

Sitio del estudio. Los tractos reproductivos recuperados para el estudio provinieron de ganado del municipio de Montería, el cual presenta topografía semiondulada con alturas que oscilan entre los 13 y $40 \mathrm{msnm}$. La precipitación anual fluctúa entre 900 y $1300 \mathrm{~mm}$ con humedad relativa del $85 \%$ y temperatura anual de $29^{\circ} \mathrm{C}$.

Muestreo de tractos reproductivos. De todos los tractos reproductivos inspeccionados, se seleccionaron 528 , de ellos fueron 264 gestantes y 264 no gestantes. Se tuvo en cuenta que los tractos provinieran exclusivamente de hembras cebuínas multíparas, motivo por el cual estas fueron identificadas y evaluadas en los corrales antes de su sacrificio. Los tractos fueron seleccionados mediante inspección visual en la línea de evisceración; para los gestantes se tuvo en cuenta aquellos con gestación evidente entre los 2 y los 7 meses aproximadamente. Para los no gestantes se tuvo en cuenta que en uno de los ovarios hubiera un cuerpo lúteo bien desarrollado. El periodo de recolección de las muestras se extendió por 90 días aproximadamente.

Procedimientos para la recolección de la información y mediciones ováricas. Después de seleccionado cada tracto reproductivo, se separaron de ellos los ovarios y se identificaron los derechos e izquierdos. Asimismo se identificó su procedencia (vacas gestantes o vacas no gestantes). Los pesajes y mediciones de los ovarios fueron realizados en el frigorífico.

Determinación de la posición del cuerpo lúteo en el ovario. Para determinar la posición del cuerpo lúteo en el ovario, este último, y con el fin de precisar la descripción de cualquier área del ovario, se dividió en dos polos(anterior y posterior), dos bordes (fijo y libre) y dos caras (superior e inferior) (Figura 1). La posición fue dibujada en un formulario previamente diseñado teniendo en cuenta los planos anatomo-espaciales relacionados con las estructuras adyacentes y con la orientación del eje longitudinal de la gónada con relación a la línea media del animal de acuerdo con los planos propuestos por (ZEMJANIS, 1980) (Figura 2).

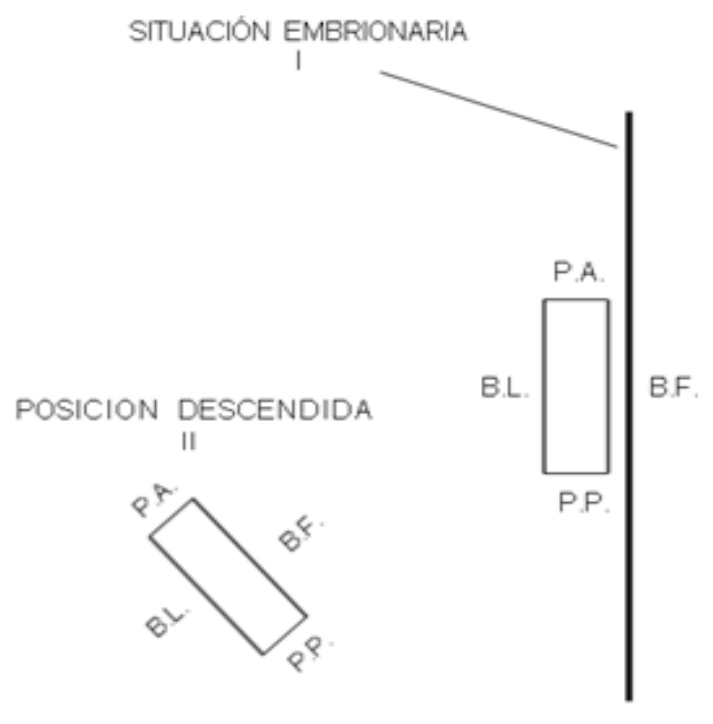

Figura 1. Esquema de los cambios de orientación del eje longitudinal del ovario con relación a la línea media del animal (línea gruesa a la derecha). I, ovario en situación embrionaria. II, Ovario en posición descendida. B.L., borde libre; B.F., borde fijo (hilio); P.A., polo anterior; P.P., polo posterior. Tomado de ZEMJANIS (1980).

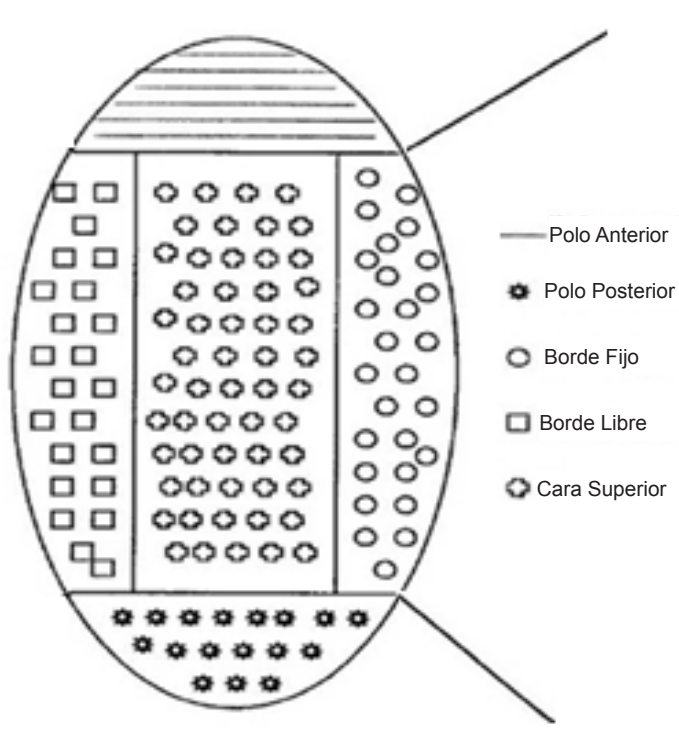

Figura 2. División anatómica del ovario (Vista cenital) para la ubicación del cuerpo lúteo en el ovario. La cara inferior corresponde a la misma área de la cara superior pero ubicada en la parte inferior del ovario. 
Medición y pesaje de los ovarios. Para establecer el volumen aproximado de los ovarios y teniendo en cuenta su forma elipsoidal se aplicó la fórmula del elipsoide en donde toda sección cortada por un plano es una elipse o un círculo. El elipsoide tiene tres ejes de simetría. En coordenadas cartesianas tridimensionales, la ecuación de un elipsoide de centro en el origen es:

$x^{2} / a^{2}+y^{2} / b^{2}+z^{2} / c^{2}=1$

La ilustración de los ovarios y de la ubicación del cuerpo lúteo se llevó a cabo en un formulario de información, en donde se consignaron los datos de pesaje tanto de los ovarios como del cuerpo lúteo. Utilizando un Nonio de Vernier se midieron los ovarios y los cuerpos lúteos teniendo en cuenta el largo, ancho y grueso. Para hallar los volúmenes del ovario y del cuerpo lúteo se utilizó la fórmula matemática descrita por DAINTITH (1982) para el elipsoide donde:

$V=a \times b \times h \times 3.1416927$

$a=\operatorname{largo}$

$\mathrm{b}=$ ancho

$\mathrm{h}=$ altura $\mathrm{o}$ grosor

Se hallaron los promedios y la desviación estándar del peso y volumen de los ovarios (izquierdo y derecho) provenientes de las vacas gestantes y no gestantes. Se utilizó la prueba de Chi Cuadrado para determinar la significancia estadística entre los datos consignados como frecuencia, tales como la determinación de la posición anatómica y espacial en vacas gestantes y no gestantes. También se aplicó la prueba "t" de student para la comparación de medias para poblaciones independientes en el caso de los pesos y volúmenes del ovario y del cuerpo lúteo, así como el peso y volumen del ovario con cuerpo lúteo y del ovario sin cuerpo lúteo, peso y volumen del cuerpo lúteo gestacional y peso y volumen del cuerpo lúteo de vacas no gestantes.

\section{Resultados}

Teniendo en cuenta que los cuerpos lúteos pueden o no sobresalir del estroma ovárico, estos, para este estudio se clasificaron en dos grupos: a) cuerpos lúteos con "corona" que corresponde a aquellos que sobresalieron en mayor o menor grado de la superficie ovárica, y b) cuerpo lúteos "internos" que fueron aquellos que no presentaron ninguna protrusión sobre la superficie ovárica. Teniendo en cuenta la clasificación anterior, a continuación se presentarán las ubicaciones de los cuerpos lúteos con "corona" tanto en vacas gestantes como no gestantes.
De las frecuencias totales de ubicación de los cuerpos lúteos con corona en el ovario de vacas gestantes (Tabla 1 ), independientemente del lado (izquierdo o derecho) y de mayor a menor fue: polo anterior $46.4 \%$ (78/168), borde libre $17.3 \%(29 / 168)$, cara inferior $16.7 \%(28 / 168)$, polo posterior $10.7 \%(18 / 168)$ y cara superior $8.9 \%$ $(15 / 168)(p \leq 0,01)$.

Tabla 1. Ubicación ovárica de los cuerpos lúteos con corona en vacas gestantes.

\begin{tabular}{cccc}
\hline $\begin{array}{c}\text { Ubicación } \\
\text { ovárica }\end{array}$ & $\begin{array}{c}\text { CL en el Ovario } \\
\text { Derecho }\end{array}$ & $\begin{array}{c}\text { Cl en el Ovario } \\
\text { Izquierdo }\end{array}$ & $\begin{array}{c}\text { Total ubicación de los } \\
\text { cuerpos lúteos }\end{array}$ \\
\hline Borde libre & $19.0 \%$ & $12.7 \%^{*}$ & $17.3 \%$ \\
& $(23 / 121)$ & $(6 / 47)$ & $(29 / 168)$ \\
Polo anterior & $44.6 \%$ & $51.1 \%$ & $46.4 \%$ \\
& $(54 / 121)$ & $(24 / 47$ & $(78 / 168)$ \\
Polo posterior & $12.3 \%$ & $6.4 \%^{*}$ & $10.7 \%$ \\
& $(15 / 121)$ & $(3 / 47)$ & $(18 / 168)$ \\
Cara inferior & $16.5 \%$ & $17.0 \%$ & $16.7 \%$ \\
& $(20 / 121)$ & $(8 / 47)$ & $(28 / 168)$ \\
Cara superior & $7.4 \%$ & $12.7 \% *$ & $8.9 \%$ \\
\hline \multirow{2}{*}{ Total } & $(9 / 121)$ & $(6 / 47)$ & $(15 / 168)$ \\
\hline & $(12.0 \%$ & $28.0 \% *$ & 168 \\
\hline
\end{tabular}

${ }^{*}(p \leq 0,01)$

De las frecuencias totales de ubicación de los cuerpos lúteos con corona en el ovario de vacas no gestantes (Tabla 2), independientemente del lado (izquierdo o derecho) y de mayor a menor fue: polo anterior $41.7 \%$ $(91 / 218)$, borde libre $23.3 \%(51 / 218)$, cara inferior $16.0 \%$ $(35 / 218)$, cara superior $11.4 \%(25 / 218)$ polo posterior $7.3 \%(16 / 218)$ y cara superior $11.4 \%(25 / 218)(p \leq 0,01)$.

Tabla 2. Ubicación ovárica de los cuerpos lúteos con corona en vacas no gestantes.

\begin{tabular}{cccc}
\hline $\begin{array}{c}\text { Ubicación } \\
\text { ovárica }\end{array}$ & $\begin{array}{c}\text { CL en el Ovario } \\
\text { Derecho }\end{array}$ & $\begin{array}{c}\text { Cl en el Ovario } \\
\text { Izquierdo }\end{array}$ & $\begin{array}{c}\text { Total ubicación de los } \\
\text { cuerpos lúteos }\end{array}$ \\
\hline Borde libre & $23 \%$ & 24.25 & $23.3 \%$ \\
& $(34 / 148)$ & $(17 / 70)$ & $(51 / 218)$ \\
Polo anterior & $41.2 \%$ & $42.8 \%$ & $41.7 \%$ \\
& $(61 / 142)$ & $(30 / 70)$ & $(91 / 218)$ \\
Polo posterior & $8.7 \%$ & $4.2 \% \%^{*}$ & $7.3 \%$ \\
& $(13 / 142)$ & $(3 / 70)$ & $(16 / 218)$ \\
Cara inferior & $16.2 \%$ & $15.7 \%$ & $16.0 \%$ \\
& $(24 / 148)$ & $(11 / 70)$ & $(35 / 218)$ \\
Cara Superior & $10.8 \%$ & $12.8 \%$ & $11.4 \%$ \\
& $(16 / 148)$ & $(9 / 70)$ & $(25 / 218)$ \\
\hline \multirow{2}{*}{ Total } & $67.9 \%$ & $32.1 \%{ }^{*}$ & 218 \\
\hline
\end{tabular}

* $(p \leq 0,01)$

De las frecuencias de la ubicación observadas de los cuerpos lúteos en los ovarios de las vacas gestantes y no gestantes, el $68.6 \%(121 / 168)$ se encontraron en el ovario derecho y el $31.4 \%(47 / 168)$ en el ovario izquierdo (Tabla 3$)(p \leq 0,01)$. 
Tabla 3. Frecuencia de cuerpos lúteos derechos e izquierdos en vacas gestantes y no gestantes

\begin{tabular}{|c|c|c|c|c|c|c|}
\hline \multirow[b]{2}{*}{ Ovarios } & \multicolumn{2}{|c|}{ Vacas gestantes } & \multicolumn{2}{|c|}{ Vacas no gestantes } & \multirow{2}{*}{$\begin{array}{l}\text { Total } \\
\text { Cuerpos } \\
\text { lúteos }\end{array}$} & \multirow[b]{2}{*}{$\%$} \\
\hline & $\begin{array}{l}\text { Cuerpos } \\
\text { lúteos }\end{array}$ & $\%$ & $\begin{array}{l}\text { Cuerpos } \\
\text { lúteos }\end{array}$ & $\%$ & & \\
\hline Derecho & 185 & 70.1 & 177 & 67.0 & 362 & $68.6^{*}$ \\
\hline Izquierdo & 79 & 29.9 & 87 & 33.0 & 166 & $31.4^{\star *}$ \\
\hline Total & 264 & & 264 & & 528 & \\
\hline
\end{tabular}

De las frecuencias de los cuerpos lúteos provenientes de vacas gestantes y no gestantes con "corona" y cuerpos lúteos "internos", el 73\% presentaron "corona" $y$ el $27 \%$ fueron "internos". ( $p \leq 0,01)$.

Tabla 4. Proporción de cuerpos lúteos con corona e internos en vacas gestantes y no gestantes.

\begin{tabular}{ccc}
\hline $\begin{array}{c}\text { Total de cuerpos lúteos en vacas } \\
\text { gestantes y no gestantes }\end{array}$ & $\begin{array}{c}\text { Cuerpos lúteos } \\
\text { con corona }\end{array}$ & $\begin{array}{c}\text { Cuerpos lúteos } \\
\text { Internos }\end{array}$ \\
\hline 528 & $73 \%(386 / 528)^{*}$ & $27 \%(142 / 528)^{* *}$ \\
\hline${ }^{*}(p \leq 0,01)$ & &
\end{tabular}

La comparación del peso y volumen del cuerpo lúteo de vacas gestantes y no gestantes se muestra en la tabla 5. El análisis estadístico para estos promedios demostró que son estadísticamente significativos ( $p \leq 0.05)$.

Tabla 5. Peso y volumen del cuerpo lúteo de vacas gestantes y no gestantes.

\begin{tabular}{cccc}
\hline Procedencia del cuerpo lúteo & N & Peso $(\mathbf{g})$ & Volumen $(\mathbf{c c})$ \\
\hline Vacas gestantes & \multirow{2}{*}{264} & $3.6 \mathbf{a}$ & $4.4 \mathbf{a}$ \\
& & \pm 1.4 & \pm 1.6 \\
Vacas no gestantes & 264 & $2.5 \mathbf{b}$ & $3.1 \mathbf{b}$ \\
& & \pm 1.2 & \pm 1.3 \\
\hline
\end{tabular}

Promedios sobrescritos con letras minúsculas distintas son estadísticamente diferentes $(p \leq 0.05)$

El peso del cuerpo lúteo de vacas gestantes fue un $30.6 \%$ más pesado que el cuerpo lúteo de vacas no gestantes. Consecuentemente, el volumen del cuerpo lúteo de vacas gestantes fue mayor en un $29.6 \%$ que el volumen del cuerpo lúteo de vacas no gestantes.

Tabla 6. Peso y volumen de los ovarios con y sin cuerpo lúteo de vacas gestantes y no gestantes.

\begin{tabular}{|c|c|c|c|c|c|}
\hline \multirow[b]{2}{*}{ Clasificación } & \multirow[b]{2}{*}{$\mathbf{N}$} & \multicolumn{2}{|c|}{ Vacas gestantes } & \multicolumn{2}{|c|}{ Vacas no gestantes } \\
\hline & & Peso (g) & Volumen (cc) & Peso (g) & Volumen (cc) \\
\hline $\begin{array}{l}\text { Ovarios con } \\
\text { cuerpos lúteos }\end{array}$ & 264 & $\begin{array}{l}11.1 \mathrm{a} \\
\pm 3.8\end{array}$ & $\begin{array}{l}12.6 \mathbf{a} \\
\pm 5.1\end{array}$ & $\begin{array}{l}10.1 \mathrm{a} \\
\pm 4.4\end{array}$ & $\begin{array}{l}11.2 \mathrm{a} \\
\pm 5.0\end{array}$ \\
\hline $\begin{array}{l}\text { Ovarios sin } \\
\text { cuerpos lúteos }\end{array}$ & 264 & $\begin{array}{l}6.4 \mathrm{~b} \\
\pm 4.6\end{array}$ & $\begin{array}{l}6.8 \mathbf{b} \\
\pm 3.4\end{array}$ & $\begin{array}{l}6.3 \mathbf{b} \\
\pm 3.8\end{array}$ & $\begin{array}{l}6.6 \mathrm{~b} \\
\pm 4.4\end{array}$ \\
\hline
\end{tabular}

Promedios sobrescritos con letras minúsculas distintas son estadísticamente diferentes $(p \leq 0.05)$.
La proporción de cuerpos lúteos quísticos en vacas gestantes y no gestantes fue de 16.3 y $12.9 \%$ y no fue significativa $(p \geq 0.05)$.

Tabla 7. Proporción de cuerpos lúteos quísticos en vacas gestantes y no gestantes.

\begin{tabular}{cccc}
\hline Procedencia & N & $\begin{array}{c}\text { Cuerpos lúteos } \\
\text { quísticos }\end{array}$ & $\%$ \\
\hline Vacas gestantes & 264 & 43 & $16.3^{*}$ \\
Vacas no gestantes & 264 & 34 & $12.9^{*}$ \\
\hline
\end{tabular}

$*(p \geq 0.05)$. No significativo.

De las frecuencias de observación de las formas del cuerpo lúteo en vacas gestantes y no gestantes se encontró que el $71.2 \%$ de ellos presentaron forma ovalada, el $25 \%$ forma piramidal y el $3.8 \%$ presentaron forma redondeada.

Tabla 8. Forma del cuerpo lúteo en los ovarios de vacas gestantes y no gestantes.

\begin{tabular}{|c|c|c|c|c|}
\hline \multirow{2}{*}{$\begin{array}{c}\text { Forma del } \\
\text { cuerpo lúteo }\end{array}$} & \multicolumn{2}{|l|}{ Vacas gestantes } & \multicolumn{2}{|c|}{ Vacas no gestantes } \\
\hline & $\begin{array}{l}\text { Número de cuerpos } \\
\text { lúteos }\end{array}$ & $\%$ & $\begin{array}{l}\text { Número de cuerpos } \\
\text { lúteos }\end{array}$ & $\%$ \\
\hline Ovalada & 181 & 68.6 & 188 & $71.2^{\star \star *}$ \\
\hline Piramidal & 49 & 18.6 & 66 & $25.0^{* *}$ \\
\hline Redondeada & 34 & 12.8 & 10 & $3.8^{*}$ \\
\hline Total & 264 & 100 & 264 & 100 \\
\hline
\end{tabular}

$(p \leq 0.05)$.

\section{Discusión}

En la literatura consultada no se encontraron datos acerca de trabajos relacionados con la ubicación del cuerpo lúteo en el ovario de vacas gestantes y no gestantes; por tal razón, la discusión de este trabajo se realizará con el fin de entregar los resultados hallados y se apoyará con antecedentes que guarden alguna relación con el tema.

La ubicación anatómica del cuerpo lúteo en el ovario de las vacas gestantes y no gestantes predominante fue la del polo anterior: Le siguieron el borde libre, la cara inferior, el polo posterior y la cara superior. En el borde fijo no se halló ningún cuerpo lúteo ya que en este sitio no ocurre la ovulación.

En la literatura no se encontró ningún antecedente para comparar y discutir estos resultados.

La presentación de cuerpos lúteos internos fue del $27.0 \%$, resultado que es inédito ya que en la literatura no reporta ningún hallazgo similar. Este resultado es 
importante para los veterinarios dedicados a la práctica y tengan en cuenta que no todos los cuerpos lúteos presentan corona como generalmente son ilustrados en los textos. La presentación de los cuerpos lúteos en los ovarios derecho e izquierdo de las vacas gestantes y no gestantes fue de $68.6 \%$ y $31.4 \%$ respectivamente. Estos resultados son similares a los reportados González y col (2014) quienes encontraron una proporción de gestación derecha e izquierda de 63.1 y $36.9 \%$ en 1534 animales. Estos mismos autores también estudiaron 300 tractos reproductivos de vacas gestantes provenientes de frigorífico y determinaron que el $67.3 \%$ de la gestaciones fue del lado derecho y el $32.7 \%$ del lado izquierdo. Como estos autores no encontraron gestaciones contralaterales a la ubicación del cuerpo lúteo, se asumió que las gestaciones derechas e izquierdas cursaron también con cuerpos lúteos ipsilaterales. A su vez son similares a los encontrados por Mc DONALD (1981), quien afirma que las preñeces se derivan en un $60-85 \%$ en el cuerno derecho y un $35-40 \%$ en el cuerno izquierdo. VATTI (1992), por su parte, afirma que el ovario derecho es más activo que el ovario izquierdo en el ganado bovino.

El peso promedio de los ovarios en las 264 vacas gestantes fue de $9.7 \mathrm{~g}$ para el ovario derecho y de 7.8 g para el ovario izquierdo. Asimismo para las 264 vacas vacías fue de $9.1 \mathrm{~g}$ para el ovario derecho y $7.3 \mathrm{~g}$ para el ovario izquierdo. Por otra parte, BONADONNA (1961), informa que los ovarios de la vaca tienen un peso que oscila entre 14 y $17 \mathrm{~g}$. Esta diferencia es probable que se deba a que este autor utilizó vacas Bos taurus mientras que las de este estudio fueron Bos indicus.

ARTHUR (1991), comunicó que el tamaño y peso del ovario depende fundamentalmente, del periodo del ciclo estral en que se realice el procedimiento y de si contiene o no un cuerpo lúteo activo. El reporta datos muy parecidos a los encontrados en este trabajo en donde el ovario con cuerpo lúteo pesó $11.1 \mathrm{~g}$ y tuvo un volumen de $12.6 \mathrm{cc}$ con respecto al ovario sin cuerpo lúteo cuyo peso fue de $6.4 \mathrm{~g}$ y el volumen de $6.8 \mathrm{cc}$ para las vacas gestantes. De la misma manera para las vacas no gestantes, el peso del ovario con cuerpo lúteo fue de $10.1 \mathrm{~g}$ y el volumen de $11.2 \mathrm{cc}$, con respecto al ovario sin cuerpo lúteo cuyo peso fue de $6.3 \mathrm{~g}$ y el volumen fue de $6.6 \mathrm{cc}$.

FRANDSON y SPURGEON (1995), afirman que el aumento del peso del cuerpo lúteo es rápido. En la vaca el peso y contenido de progesterona del cuerpo lúteo aumenta rápidamente entre el tercero y décimo segundo día del ciclo. Estos hallazgos tienen alguna relación con los resultados de este trabajo donde el peso del cuerpo lúteo gestacional es de $3.6 \mathrm{~g}$ y el volumen de $4.4 \mathrm{cc}$ con respecto al peso del cuerpo lúteo cíclico (vacas no gestantes) que es de $2.5 \mathrm{~g}$ y el volumen de $3.1 \mathrm{cc}$. Por otra parte, ZEMJANIS (1980), comunica que los cuerpos lúteos quísticos se observan frecuentemente en el post-parto de las vacas y no difiere con la función reproductiva del animal. En este trabajo no se encontró ninguna diferencia entre los porcentajes de los cuerpos lúteos quísticos de las vacas gestantes (16.3\%) y vacías (12.9\%).

La proporción de la forma del cuerpo lúteo en los ovarios de las vacas gestantes fue de $68.5 \%$ ovalada, $18.5 \%$ piramidal, $12.8 \%$ redondeada, para las vacas vacías es de $71.2 \%$ ovalada, $25 \%$ piramidal, $3.7 \%$ redondeada, no se halló algún dato referente a la forma del cuerpo lúteo en la literatura. Cabe mencionar que de acuerdo con el sitio de ovulación y forma que tome el cuerpo lúteo, afectará la forma que tome el ovario y que será la que finalmente se palpe.

ARTHUR (1991), informó que el caso de contralateralidad ocurría con una incidencia del $1 \%$. Perkins et al, citado por ROBERTS (1979), señala que menos del $2 \%$ de las gestaciones tenían un cuerpo lúteo en el ovario contralateral al cuerno preñado. Erdheim, citado por ROBERTS (1979), informó un caso en 3824 vacas. ROBERTS (1979), lo observaron en un $0.03 \%$ de cada 4000 casos, contrario a lo hallado por González y col (2014), que no reportaron ningún caso en 1836 animales, dato que concuerda con lo hallado en este trabajo donde en 528 vacas no se reportó ningún caso de gestación contralateral a la ubicación del cuerpo lúteo.

\section{Conclusiones}

Se determinó que la posición anatómica del cuerpo lúteo en los ovarios de las vacas gestantes y vacías se da con mayor frecuencia en el polo anterior, le siguen en su orden el borde libre, cara inferior, polo posterior, cara superior. En ambos casos no se encontró ningún cuerpo lúteo en el borde fijo. Se estableció la presencia de cuerpos lúteos interno. Se confirmó que el ovario derecho es más activo que el ovario izquierdo y por lo tanto su peso y volumen son mayores. Se estableció que el peso y volumen del cuerpo lúteo gestacional es mayor que el cuerpo lúteo cíclico. 


\section{Referencias}

ARTHUR, GEOFFREY; NOAKES, DAVID y PEARSON, HAROLD. 1991. Reproducción y obstetricia en veterinaria. 6 ed. España: Interamericana; p:19-22.

BONADONNA, T. 1961. Fisiopatología de la reproducción y de la fecundación artificial de los animales domésticos. Barcelona: Salvat; p:304-307.

DAINTITH, JOHN. 1982. Diccionario de matemáticas. Bogotá: Editorial Norma; p:72-73.

DERIVAUX, J. 1961. Fisiopatología de la reproducción e inseminación artificial de los animales domésticos. Zaragoza: Acribia; p:4-9.

FRANDSON, R.D. y SPURGEON, T.L. 1995. Anatomía y fisiología de los animales domésticos. 5 ed. México: Interamericana; p:421-423.

GARCIA SACRISTAN, 1995. A Fisiología veterinaria. España: Interamericana- Mc Graw-Hill; p:931-933.

GONZALEZ, T.M; PASTRANA, PN; BARÓN, P.F; VERTEL, M.M. 2014. Frecuencia de presentación de gestacion con relación al cuerno uterino en bovinos del trópico colombiano. Rev Med Vet (28):13-21.

HANSEL, WILLIAM, M. and BLAIR, ROBERT. 1996. Bovine corpus luteum: A historie overview and implications for future reserach. Theriogenology 45:1267-1277.

HOLY, LUBOS. 1987. Bases biológicas de la reproducción bovina. México: Diana: p:59-61.

Mc DONALD, LE. 1981. Reproducción y endocrinología veterinarias. 2 ed. México: Interamericana; p:277.

ROBERTS, STEPHEN. 1979. Obstetricia veterinaria y patología de la reproducción teriogenología. Buenos Aires: Hemisferio Sur: p:624-642.

VATTI, GIUSEPPE. 1992. Ginecología y obstetricia veterinaria.Mexico: Limusa; p:130.

ZEMJANIS, R. 1980 Reproducción animal, diagnóstico y técnicas terapéuticas. México: Limusa; p:82-88. 